\title{
Outcome of the first Saudi Central Board for Accreditation of Healthcare Institutions (CBAHI) primary health care accreditation cycle in Saudi Arabia
}

\author{
Maher A. Alsakkak, MD,SBFM, Salem A. Alwahabi, MD, SBGS, Hamad M. Alsalhi, MD, DLO, Majdah A. Shugdar, DBA,CPHQ.
}

\begin{abstract}
الأهداف : دراسة وتقييم المرحلة الأولى من دورة اعتماد سيباهي المرادي لمراكز الرعاية الصحية الأولية.

الطريقة: دراسة تحليلية وصفية لتقييم 93 زيارة استقصائية لمراكز

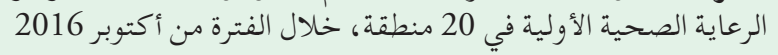
إلى مايو 2017.

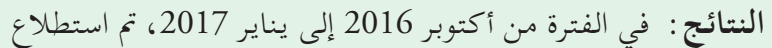

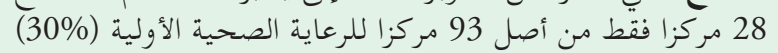

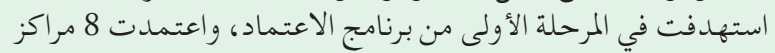

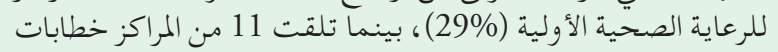

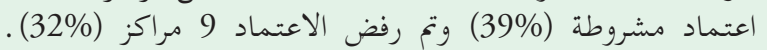

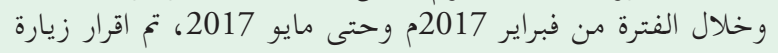

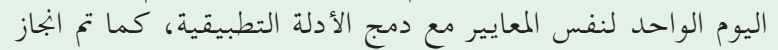

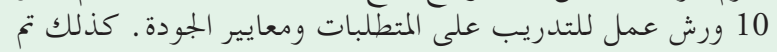

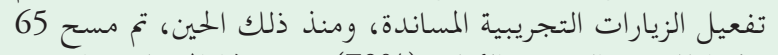

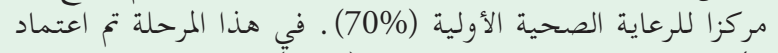

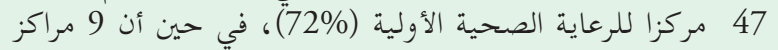

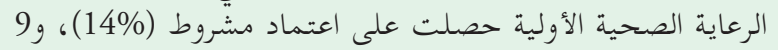

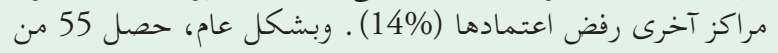

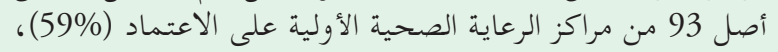

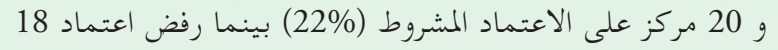

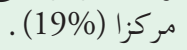

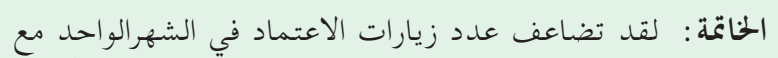

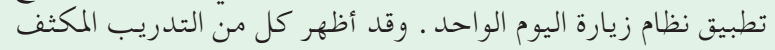

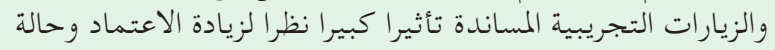

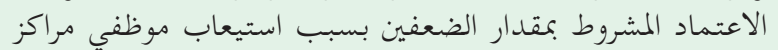

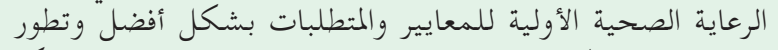

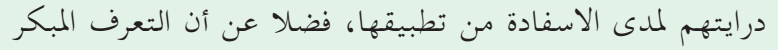

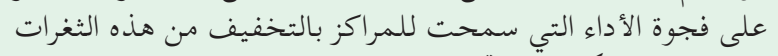

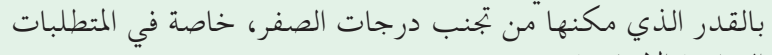

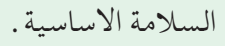

Objectives: To study and evaluate the first phase of the Saudi Central Board for Accreditation of Health Institutions (CBAHI) in primary health care (PHC) accreditation cycle.
Methods: A descriptive analytical study of to evaluate 93 PHC survey visits in 20 regions, over the period October 2016 to May 2017.

Results: In the period October 2016 to January 2017 , only 28 out of 93 PHC center $(30 \%)$ targeted PHC in phase- 1 were surveyed, 8 PHC got accredited (29\%), while 11 PHC received conditional accreditation letters (39\%) and 9 PHC were denied the accreditation (32\%). During February 2017 to May 2017 visits were shifted to one day site visit, all the health care facilities training's workshops were completed and the mock survey visits were started to precede the real survey visits, Since then, 65 PHC centers were surveyed (70\%). At this time 47 PHC centers were accredited (72\%), while 9 PHC were conditionally accredited (14\%), and another 9 PHC were denied the accreditation (14\%). Overall, 55 out of 93 PHC accredited (59\%), 20 PHC got conditional accreditation (22\%), and 18 PHC were denied the accreditation (19\%).

Conclusion: Surveys per month were doubled with the one-day visit site visit. Both the intensive training and mock visits, showed a great impact as the accreditation and conditional accreditation status were increased by twofold due to better understanding of the PHC staff about the standards, as well as early identification of performance gap which allowed the PHC to mitigate these gaps sufficiently enough to avoid zero scores especially in the ESR requirements.

Saudi Med J 2017; Vol. 38 (11): 1132-1136 doi: 10.15537/smj.2017.11.20760

From the Primary Health Care Accreditation Unit (Alsakkak, AlWahabi, Shugdar), and the Research \& Development (Alsalhi), Saudi Central Board for Accreditation of Health Institutions, Riyadh, Kingdom of Saudi Arabia.

Received 22nd July 2017. Accepted 9th August 2017.

Address correspondence and reprint request to: Dr. Maher Alsakkak, Primary Health Care Accreditation Unit, Saudi Central Board for Accreditation of Health Institutions, Riyadh, Kingdom of Saudi Arabia.E-mail:masakkak@cbahi.gov.sa

ORCID ID: orcid.org/0000-0002-5615-4098 
$\mathrm{I}$ $\mathrm{n}$ the dawn of the evidence-based health practice and information technology era, healthcare institutions struggle with paradoxes of vast varieties. ${ }^{1}$ They need to focus on multiple goals, such as medical education and patients' health care through different strategic axes like managerial, professional, technocratic, and others. ${ }^{2,3}$ They must allow the medical staff to exercise their clinical judgments while promoting the standardization of practices. ${ }^{4}$ They must act autonomously and innovate, in coordination with other related health providers, to meet their goals and the community expectations. This has been established to increase pressure to improve performance, as many publications have reported serious limitations in the quality and safety of health care practices. ${ }^{5}$ One important mean of tackling this burden is accreditation. Accreditation for healthcare institutions involves an accrediting body surveying and verifying compliance with recognized criteria/standards. ${ }^{6}$ It has been considered as a useful tool to establish national standards and reduce variations in medical practice, ${ }^{7}$ it has been shown to mitigate inappropriate medical care $^{8}$ and improve the cost-effectiveness especially in with strict and recessive economy. One study showed that almost $95 \%$ of contracted services made by health care governing companies in the United States are with accredited health care facilities. ${ }^{9}$ This demonstrates that accreditation status of health care facilities plays a major role in the health care clients' decisions. Failure of an organization to go through an accreditation process may indicate that the facility is not open to external evaluation of its performance. ${ }^{10}$

There are many international accrediting bodies to evaluate the quality and patient's safety in health care institutions, yet the Saudi Central Board for Accreditation of Healthcare Institutions (CBAHI) is the only national agency authorized to grant accreditation certificates to all governmental and private healthcare facilities operating in Saudi Arabia. CBAHI has emerged from the Saudi Health Council as a non-profit organization. ${ }^{11}$ The principal function of CBAHI is to set the healthcare quality and patient safety standards against which all healthcare facilities are evaluated for evidence of compliance.

The Primary health care centers' general directorate of the ministry of health in Saudi Arabia decided to utilize CBAHI to evaluate 2386 Primary Health care

Disclosure. Authors have no conflict of interests, and the work was not supported or funded by any drug company.
(PHC) centers affiliated to its own administration aiming to ensure high-quality standards and safe health care environment. The general directorate of $\mathrm{PHC}$ centers in $\mathrm{MOH}$ selected 502 PHC centers in 20 regions as a target in the period between October 2016 and May 2020 in 4 consecutive phases. The aim of this descriptive analytical study is to highlight the result of the 1st phase of CBAHI PHC accreditation cycle in Saudi Arabia.

The objective of this study is to highlight the results of the 1st phase of CBAHI PHC accreditation cycle in Saudi Arabia, including the accreditation decision status, the impact of 3 mid-cycle interventions, and the most scored essential safety requirements (ESR) standards.

Methods. A descriptive analytical study that involved 93 PHC centers visited for survey in 20 regions within Kingdom of Saudi Arabia (KSA) to evaluate their compliance with national primary health care standards by CBAHI, over the period from October 2016 to May 2017. It represents the 1st CBAHI accreditation cycle of the PHC facilities affiliated to the ministry of health in KSA. Visits were conducted by 28 surveyors who received intensive cross training all over the 23 chapters to evaluate Leadership, Manpower, Management of Information, Quality Management and Patient Safety, General clinics, referrals, Health Record, Dental and Oral Health, Patient and Family Rights, Health Promotion and Education, Radiological Services, Maternal and Child Health, Immunization, Communicable Diseases, Non-Communicable Diseases, Geriatric Care, Environmental Health, Emergency Services, Community Participation, Facility Management and Safety, Infection Prevention and Control, Laboratory Services, and Pharmaceutical Services.

Each survey visit was commenced by 2 surveyors, over 1-2 days' survey agenda. The accreditation decision was done after robust reports review by health care accreditation specialists and the PHC surveyors' team leader as well as in depth discussion by the accreditation decision committee. The accreditation decision matrix relies on three items; total score, Essential safety requirements (ESR) score, and the number of zero scored ESR. Table 1 demonstrate the cut points of the accreditation decision matrix:

In the period from October 2016 to January 2017, the survey visits were conducted over 2 days ranging from 3-9 visits per month. In the period from February 2017 to May 2017, visits were only for one day through merging of evidence of compliance without any modification in the standards and/or the sub-standards 
Table 1 - Central Board for Accreditation of Healthcare Institutions (CBAHI) primary health care Accreditation Decision Matrix.

\begin{tabular}{lccccc}
\hline Total score & + & ESR score & + & $\begin{array}{c}\text { Zero scored } \\
\text { ESR }\end{array}$ & Decision \\
\hline$\geq 85 \%$ & and & $\geq 85 \%$ & and & 0 & Accredited \\
$\geq 75$ to $<85 \%$ & or & $\geq 75$ to $<85 \%$ & or & $1-5$ & $\begin{array}{c}\text { Conditional } \\
\text { accreditation } \\
\text { Denial }\end{array}$ \\
\hline $75 \%$ & or & $<75 \%$ & or & $>5$ & DSR - essential safety requirements \\
\hline \multicolumn{5}{c}{} \\
\hline
\end{tabular}

which led to an increase of the survey visits to $12-20$ visits per month. CBAHI also exerted an effort to educate and train 500 PHC centers' staff through 10 workshops for Health care facilities orientation, aiming to improve their knowledge as well as understanding and compliance with the standards. All workshops were started by October 2016 and completed by end of February 2017. One more intervention was applied through mock survey visits by CBAHI-MOH surveyors to augment and support the standards implementation through early identification and analysis of performance gap.

Inclusion and exclusion. All the 93 PHC visits were included in the study and none were excluded.

Statistical analysis. Descriptive analytical were used to measures of central tendency frequency distribution and spread using Microsoft Excel 2016.

Results. In the period from October 2016 to January 2017, only 28 out of 93 PHC centers (30\%) targeted in phase-1 were surveyed (Figure 1). Eight PHC centers got accredited (29\%), while 11 received conditional accreditation letters (39\%) and 9 were denied the accreditation (32\%) (Figure 2).

Since February 2017 through May 2017 visits were changed to a one-day site visit. Health care facilities training workshops and the mock survey visits now preceded the actual survey visits; afterwards 65 PHC centers $(70 \%)$ were surveyed. Subsequently, 47 PHC centers were accredited (72\%), 9 were conditionally accredited (14\%), and another 9 centers were denied the accreditation (14\%) (Figure 3). Overall, 55 out of 93 PHC centers were accredited (59\%), 20 PHC got conditional accreditation (22\%), and 18 PHC were denied the accreditation (19\%) (Figure 4).

The highest total score was $98.87 \%$ and the lowest total score was $44.91 \%$; both were achieved by PHC centers in the period from February 2017 to May 2017. The lowest essential safety requirements (ESR) score was $14.29 \%$ in the period from October 2016 to January 2017. A total of 25 out of 93 PHC centers managed to get $100 \%$ score in ESR. Three ESR were scored frequently as not met (zero score). The most frequently scored ESR as not met was GC.19.EC.1 (documentation of the plan of care in the patients' health records) in 14 cases. The second most-commonly scored ESR deficiency was IPC.22.EC.5 (compliance in the central sterilization unit and sterilizations requirements) in 12 cases. The ESR of evaluation and completion of all the required items in prescriptions (PH.14.EC.2) was the third most common deficiency, in 11 cases.

Discussion. The PHC survey visits originally followed a 2-day agenda and, although the mean site visits per month was 12 , the number of visits per month did not exceed nine visits. After four months, the CBAHI primary health care team reviewed the evidence

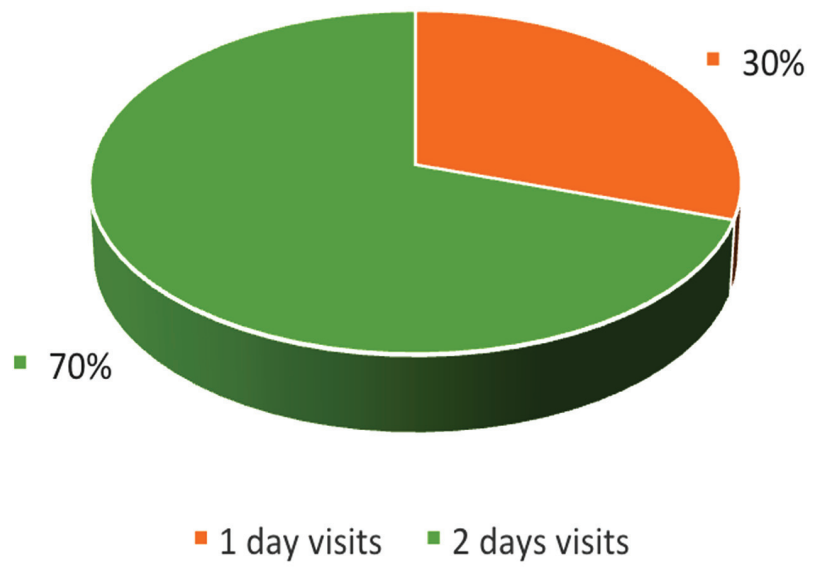

Figure 1 - Comparison between one day visit verses 2 days visits during February 2017 to May 2017.

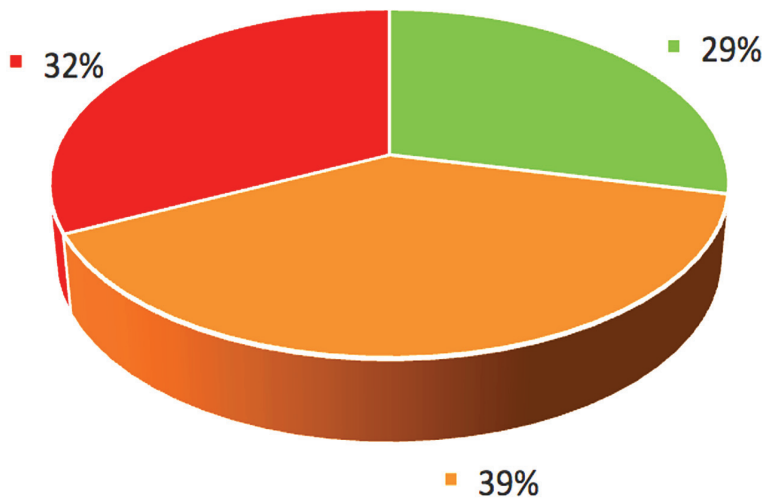

- Accredited " Conditional Accreditation - Denial

Figure 2 - Primary health care results of accredited, conditional accreditation, and denial between October 2016 and January 2017. 


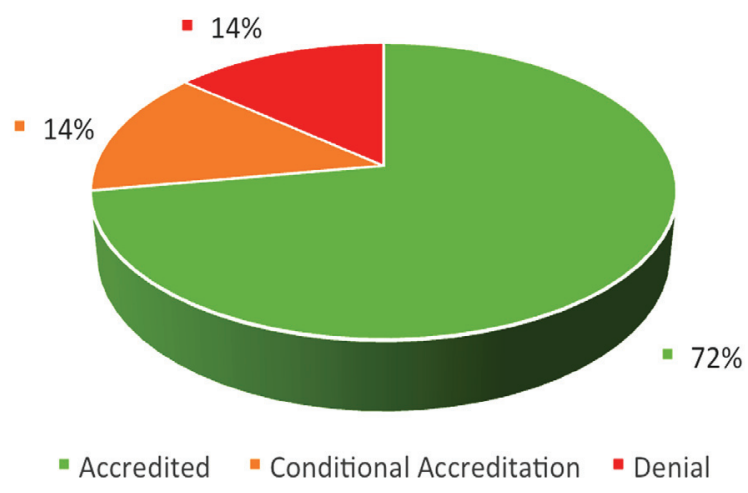

Figure 3 - Primary health care results of accredited, conditional accreditation, and denial between February 2017 and May 2017.

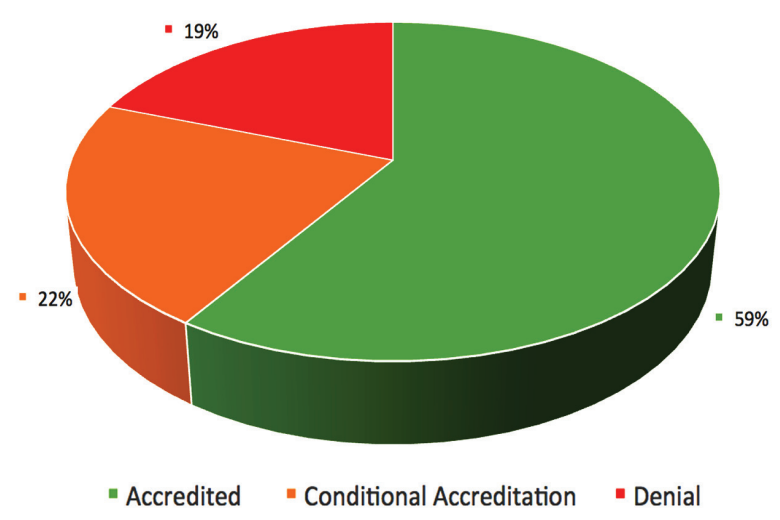

Figure 4 - Primary health care results of accredited, conditional accreditation, and denial between October 2016 and May 2017.

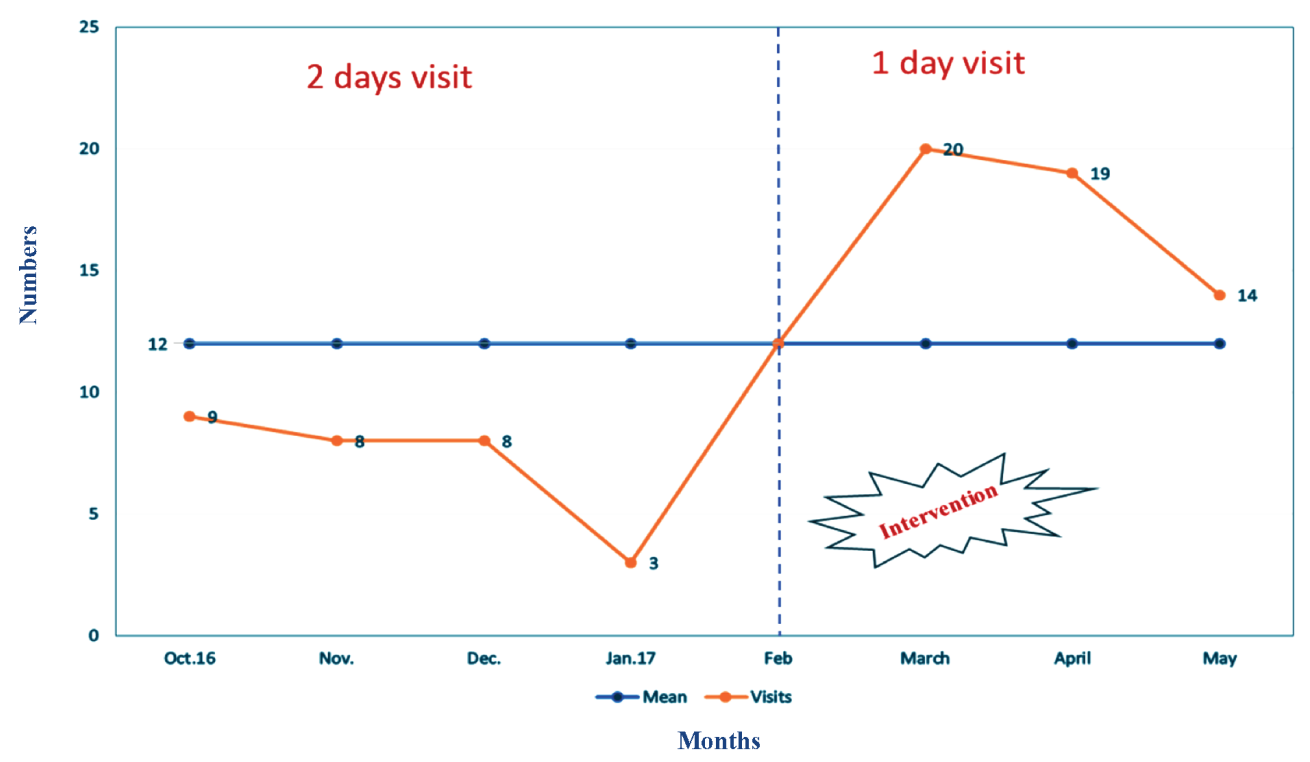

Figure 5 - Primary health care visits per month between October 2016 and May 2017.

of compliance and shortened the visits agenda without affecting the standards and sub-standards numbers or contents, through merging of evidence of compliance by focusing on the implementation rather than bulk documents review. Two pilot visits were conducted to evaluate the modification in the evidence of compliance and agenda, which showed no significant changes in survey visit accreditation status results, suggesting that shorter visits would not fail to find deficiencies due to lack of time. Thereafter, PHC centers were scheduled for one day visits, and the total number of visits per month increased to 20 visit per months (Figure 5). Clearly, the number of visits per month doubled with one day visits which could be explained by the increased availability of surveyors, who are all part-time employees of the CBAHI. The one day agenda reduced the interruption in their full-time job schedules.

Ten intensive training workshops to train 500 PHC staff from all over the 20 regions in Saudi Arabia in the targeted PHC centers were conducted through strategic collaborative relationship between CBAHI and $\mathrm{MOH}$. Each workshop was conducted by senior 
CBAHI primary health care surveyors over 3 days. The workshop was intended to improve the ability of the staff to understand the intent of the standards and how to comply with the standards requirements, as well as the quality concepts and quality tools. Post-tests were conducted to ensure that the staff accomplished the objectives of the training.

Based on the improvement in accreditation results, the mock survey visits appeared to be of great help to the PHC centers as they assisted the PHC leaders and staff to identify the gaps for improvement and helped to focus their efforts to a significant few through utilizing what they have learned in the training workshop, in accordance with "the rule of 80:20" (namely, an economic principle of 'factor sparsity' which states the as much of $80 \%$ of an effect stems from only $20 \%$ of potential causes).

One limitation in this study, is that there is no comparison with international accreditation process and it was designed as a descriptive analytical theme to describe the first CBAHI Primary Health Care Accreditation Cycle in Saudi Arabia with the impact of 3 mid-cycle intervention.

In conclusion, the change to a one-day site visit doubled the number of visits efficiently while both the intensive training and mock visits showed a great impact in the results as the accreditation and conditional accreditation status were increased by 2 fold due to better understanding of the PHC staff about the standards as well as early identification of the performance gap which allowed the PHC to mitigate these gaps sufficiently enough to avoid zero scores especially in the ESR requirements.

\section{References}

1. Pomey MP, Lemieux-Charles L, Champagne F, Angus D, Shabah A, Contandriopoulos AP. Does accreditation stimulate change? A study of the impact of the accreditation process on Canadian healthcare organizations. Implement Sci 2010; 5: 31.

2. Glouberman S, Mintzberg H. Managing the care of health and the cure of disease--Part I: Differentiation. Health Care Manage Rev 2001; 26: 56-69.

3. Glouberman S, Mintzberg H. Managing the care of health and the cure of disease--Part II: Integration. Health Care Manage Rev 2001; 26: 70-84.

4. Champagne F, Contandriopoulos AP, Denis JL, Lamothe L. Developing a conceptual model for security analysis in health organizations. Appendix report submitted to Health Canada Montreal. Montréal: GRIS, Université de Montréal; 2002.

5. World Health Organization. The world health report 2000 Health systems: improving performance. Geneva: WHO; 2000.

6. The Institute of Medicine: Committee on Quality of Health Care in America. In Crossing the Quality Chasm: A New System for the 21st Century Washington (DC): National Academy Press; 2001.

7. American Society for Quality. [Accessed 2017 June 6th]. Available from URL: https://asq.org/quality-resources/qualityglossary

8. Wennberg JE. Understanding geographic variations in health care delivery. N Engl J Med 1999; 340: 52-53.

9. Brook RH, Lohr KN. Will we need to ration effective healthcare? Issues Sci Technol 1986; 3: 68-77.

10. Bergman R. No accreditation, no contract, say most managed care plans. Hosp Health Netw 1994; 68: 64.

11. Saudi Central Board for Accreditation of Health Institutions [Updated 2008; Accessed 2017 June 21]. Available from URL: http://portal.cbahi.gov.sa/english/about-us/cbahi-at-a-glance. 